\title{
Identification of a novel AluSx-mediated deletion of exon 3 in the SBDS gene in a patient with Shwachman-Diamond syndrome
}

\author{
Elísio Costa ${ }^{\mathrm{a}}$, Frederico Duque ${ }^{\mathrm{b}}$, Jorge Oliveira ${ }^{\mathrm{c}}$, Paula Garcia ${ }^{\mathrm{b}}$, Isabel Gonçalves ${ }^{\mathrm{b}}$, \\ Luísa Diogo ${ }^{\mathrm{b}}$, Rosário Santos ${ }^{\mathrm{c}, *}$ \\ a Escola Superior de Saúde, Instituto Politécnico de Bragança, Bragança, Portugal \\ ${ }^{\mathrm{b}}$ Hospital Pediátrico de Coimbra, Coimbra, Portugal \\ ${ }^{c}$ Unidade de Genética Molecular, Instituto de Genética Médica Jacinto Magalhães, 4099-028 Porto, Portugal
}

Submitted 30 January 2007

Available online 21 March 2007

(Communicated by M.A. Lichtman, M.D., 13 February 2007)

\begin{abstract}
Shwachman-Diamond syndrome (SDS) is caused by mutations in the SBDS gene, most of which are the result of gene conversion events involving its highly homologous pseudogene $S B D S P$. Here we describe the molecular characterization of the first documented gross deletion in the $S B D S$ gene, in a 4-year-old Portuguese girl with SDS. The clinical diagnosis was based on the presence of hematological symptoms (severe anemia and cyclic neutropenia), pancreatic exocrine insufficiency and skeletal abnormalities. Routine molecular screening revealed heterozygosity for the common splicing mutation c. $258+2 \mathrm{~T}>\mathrm{C}$, and a further step-wise approach led to the detection of a large deletion encompassing exon 3 , the endpoints of which were subsequently delineated at the gDNA level. This novel mutation (c.258+374_459+250del), predictably giving rise to an internally deleted polypeptide (p.Ile87_Gln153del), appears to have arisen from an excision event mediated by AluSx elements which are present in introns 2 and 3.

Our case illustrates the importance of including gross deletion screening in the SDS diagnostic setting, especially in cases where only one deleterious mutation is detected by routine screening methods. In particular, deletional rearrangements involving exon 3 should be considered, since Alu sequences are known to be an important cause of recurrent mutations.
\end{abstract}

(C) 2007 Elsevier Inc. All rights reserved.

Keywords: Shwachman-Diamond syndrome; SBDS gene; SDS; Anemia; Neutropenia; Pancreatic insufficiency; Gross deletion

\section{Introduction}

Shwachman-Diamond syndrome (SDS; MIM \#260400) is a rare autosomal recessive disorder first described in 1964 [1]. The association of bone marrow and exocrine pancreatic dysfunctions characterizes this disease, which usually manifests clinically in infancy or early childhood. Other systemic findings (skeletal, liver and psychomotor) or problems secondary to bone marrow dysfunction may also be present [1-4]. Intermittent or persistent neutropenia, present in approximately $40 \%$ of the patients, is the most common hematological finding, but anemia and thrombocytopenia may also be found [1-4]. Diagnosis is not always obvious since mitochondrial respiratory chain (MRC)

\footnotetext{
* Corresponding author. Fax: +351 226070399.

E-mail address: rosario.santos@igm.min-saude.pt (R. Santos).
}

disorders, particularly Pearson syndrome, share most of its clinical manifestations.

The SDS locus was mapped to chromosome $7 \mathrm{q} 11$ where disease-associated mutations were later reported in the Shwachman-Bodian-Diamond syndrome gene (SBDS; MIM \#607444) [5-7]. This gene comprises five exons spanning $7.9 \mathrm{~kb}$, with a $1.6 \mathrm{~kb}$ transcript that translates into a protein of 250 amino acids $[6,7]$. The SBDS protein is a member of a highly conserved family with orthologs in several species. Although its function remains to be elucidated, studies revealing ubiquitous expression with accumulation in the nucleolus in a cell-cycledependent manner, as well as structural and co-expression studies in the yeast orthologs, provide strong evidence for its role in ribosome biogenesis [7-9].

The majority of mutations described in the $S B D S$ gene are said to represent gene conversion events due to recombination 
with its pseudogene $S B D S P$, with which it shares $97 \%$ identity [7,10-13]. Many have been found to occur within a $240 \mathrm{bp}$ region around exon 2 , which harbors the most frequent mutations c.183_184TA $>$ CT and c.258+2T $>$ C. These are often seen to be associated with the polymorphic variants c. $141 \mathrm{C}>\mathrm{T}$ and/or c. $201 \mathrm{~A}>\mathrm{G}$, presumably as a result of the size of the DNA fragments involved in the rearrangements $[7,9,10,12,13]$. However, mutations may occur throughout the gene, as evidenced by the 22 variants documented to date. Of these, $50 \%$ are missense mutations and the remainder includes splicing mutations (18\%), small deletions (14\%), del/ins conversions $(9 \%)$, small duplications $(5 \%)$ and nonsense mutations (5\%) [7,9,10,12-15]. No gross deletions have been reported in the $S B D S$ gene.

We describe the molecular characterization of a Portuguese patient with SDS, who was found to be compound heterozygous for the known mutation c. $258+2 \mathrm{~T}>\mathrm{C}$ and the hitherto undocumented in-frame deletion of exon 3 (c.258+374_459 +250del). This deletion appears to have been mediated by AluSx repetitive sequences in the flanking introns.

\section{Patient, materials and methods}

\section{Case report}

This girl was the second child of a Caucasian nonconsanguineous couple, with irrelevant family history. Her perinatal history was uneventful. She was admitted for the first time at 3 months of age with failure to thrive, severe anemia (hemoglobin: $5.6 \mathrm{~g} / \mathrm{dl}$ ), extensive eczema and liver enlargement with hepatitis. She had multiple admissions in the first year of life, for blood cell transfusions or serious clinical infections, due to cyclic neutropenia. Investigation revealed bone marrow dysfunction and persistent hepatitis.

Pearson syndrome was suspected and at 6 months of age she was subjected to a muscle biopsy (no histological abnormalities) and a liver biopsy (reactive chronic hepatitis and hemosiderosis). MRC evaluation showed a decrease in complex IV $(25 \%$ of normal control average, in relation to citrate synthase) in the muscle, and in complexes II (10\%), II/III
$(20 \%)$, IV (9\%) and V (16\%) in the liver. The mitochondrial DNA study showed no common mutations. At 14 months of age pancreatic insufficiency was evidenced (fecal elastase: $36 \mu \mathrm{g} / \mathrm{g}$; $\mathrm{N}>200 \mu \mathrm{g} / \mathrm{g}$ ) and pancreatic enzyme supplementation was introduced. At 17 months of age treatment with prednisone $(1 \mathrm{mg} / \mathrm{kg} /$ day) was started and maintained for 1 year. A second liver biopsy, after 5 months on prednisone, evidenced mild chronic hepatitis and normal activity of MRC enzymatic complexes. SDS was then considered and a skeletal survey revealed metaphyseal chondrodysplasia (Fig. 1). She is presently 5 years old. Sustained recovery from anemia and neutropenia occurred, but mild hepatitis, recurrent eczema and short stature remains. Psychomotor development is normal. Informed written consent was obtained from the parents for all molecular studies.

\section{gDNA analysis}

Genomic DNA (gDNA) of the patient and her parents was isolated from blood samples using standard methods. Direct sequencing of all coding regions of the $S B D S$ gene, including exon-intron boundaries, was performed by cycle-sequencing using polymerase chain reaction (PCR) primers as described previously $[10,16]$. For analysis of intron 2 , the following primers were designed: g.SBDSI2F: 5'CCA CTC AGT GTT TGT AAA TGT TTC TA and g.SBDSI2R: 5'TGT ATG GTC TCT TTG TTT CAG GAT. Amplification of a 1870-bp fragment corresponding to exon 3 plus a large portion of its surrounding intronic sequences was performed using the primers g.SBDS3bF: 5'TGC CCC ATT TAT AGA ATG TGG TA and g.SBDS3bR: 5' AAA GCA ACC CAA TGT CTA TCA AC. All PCR reaction mixtures contained $25 \mu \mathrm{l}$ PCR Master Mix (Promega, Madison, USA), $22 \mu \mathrm{l}$ water, $1 \mu \mathrm{l}$ of each primer (each at $25 \mathrm{pmol} / \mu \mathrm{l}$ ) and $1 \mu \mathrm{gDNA}(250 \mathrm{ng} / \mu \mathrm{l})$. Amplification cycles consisted of an initial denaturation step of $95^{\circ} \mathrm{C}$ for $10 \mathrm{~min}$, followed by 35 cycles of $95{ }^{\circ} \mathrm{C}$ for $1 \mathrm{~min}, 55^{\circ} \mathrm{C}$ for $1 \mathrm{~min}$ and $72^{\circ} \mathrm{C}$ for $2 \mathrm{~min}$, and with a final extension at $72{ }^{\circ} \mathrm{C}$ for $10 \mathrm{~min}$.

A 3662-bp fragment, ranging from the beginning of exon 2 to the end of exon 4, was amplified with primers SBDSEX2F [16] and sds4R [10], using the Expand Long Template PCR System

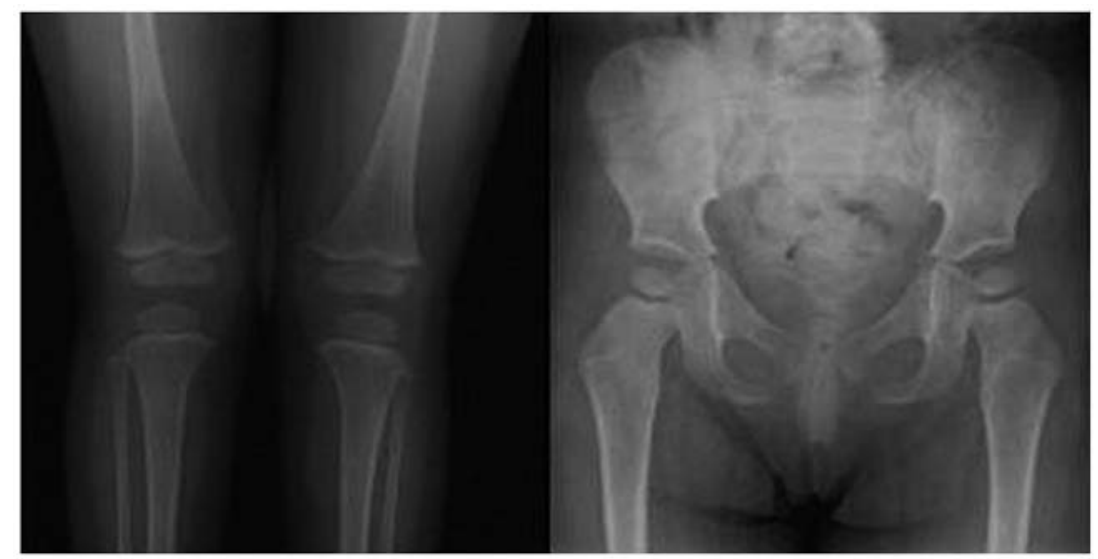

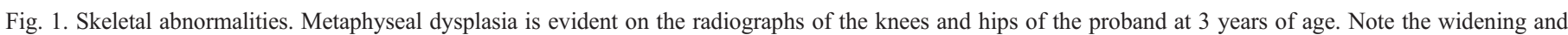
irregularity of metaphyses of the long bones and the narrowing of the sacroiliac notch. 
(Roche Diagnostics, IN, USA) in a total volume of $50 \mu \mathrm{l}$, according to the manufacturer's instructions. Thermal cycling consisted of an initial denaturation step of $95{ }^{\circ} \mathrm{C}$ for $10 \mathrm{~min}$, followed by 35 cycles of $95^{\circ} \mathrm{C}$ for $1 \mathrm{~min}, 55^{\circ} \mathrm{C}$ for $1 \mathrm{~min}$ and $72{ }^{\circ} \mathrm{C}$ for $5 \mathrm{~min}$, and a final extension at $72{ }^{\circ} \mathrm{C}$ for $30 \mathrm{~min}$. All amplification reactions were carried out in a GeneAmp PCR System 9600 thermal cycler (Applied Biosystems, Foster City, CA, USA).

For the sequencing reactions the amplicons were isolated by electrophoresis on a $2 \%$ agarose gel and then purified using spin$\mathrm{X}^{\circledR}$ (Corning Incorporated, NY, USA). The nucleotide sequence was determined using the respective forward and reverse primers, in independent sequencing reactions, with the Dye Terminator Cycle Sequencing Kit v1.1 (Perkin Elmer, Boston, MA, USA). The products were resolved on an ABI Prism 3130xl Genetic Analyser (Applied Biosystems, Foster City, CA, USA).

\section{cDNA analysis}

Total RNA was extracted from peripheral blood of the patient and a control using TRIzol isolation reagent (Invitrogen, CA, USA). Reverse transcription PCR (RT-PCR) was performed with Superscript One-step RT-PCR and Platinum Taq polymerase (Invitrogen, CA, USA) using the following 3 sets of primers: c.SBDS1F: 5'CTG AAG CTA GTG AGT CGC GG and c. SBDS1R: 5'TCA ATA AGG ATC ACG GTG TAT GG (exons 1 to 3); c.SBDS2F: 5'GGA ACA GAT GAC CAA ACT GAA ATC and c.SBDS2R: 5'CCG GGT CAA TCA GAC ATA CG (exons 2 to 5); c.SBDS3F: 5'AAG AAA AGC TCA AGC CAC TGA TC and c.SBDS3R: 5'CAG AGT AGC AAA ATG CCA $\mathrm{CTC}$ TG (exons 4 to 5). Fragment isolation and sequencing were carried out as described above.

\section{Bioinformatic tools}

Reference sequences used were GenBank NM_016038.2 for cDNA and AC079920 for gDNA of the SBDS gene, as well as NR 001588 for SBDSP. Mutation nomenclature was according to the recommendations of the Human Genome Variation Society (2005) (http://www.hgvs.org/mutnomen), using the numbering convention which assigns " 1 " to the A of the initiator ATG codon.

All of the new primers were designed so as to be $S B D S$ specific. To that end, reference sequences retrieved from the GenBank database were aligned using the ClustalX algorithm [17], and primers were chosen with the aid of Primer Express V2.0 software (Applied Biosystems, Foster City, CA, USA).

The possible involvement of cryptic splice sites was evaluated by scanning the variant sequence with GENSCAN software [18], that is designed to identify exon/intron structures (http://genes.mit.edu/GENSCAN.html). In order to detect homologous regions between introns 2 and 3, these were compared using BLAST 2 Sequences [19] (http://www.ncbi. nlm.nih.gov/blast/bl2seq/wblast2.cgi). The identification of repetitive sequences was aided by the CENSOR on-line tool [20], which screens queries against a reference collection of repeats (http://www.girinst.org/censor/index.php).

\section{Results}

Sequencing of the five exons of the $S B D S$ gene from the patient revealed only the presence of the known mutation c.258+ $2 \mathrm{~T}>\mathrm{C} 7$ (p.Cys84SerfsX3) and the c.201A $>\mathrm{G}$ polymorphism, both in heterozygosity. The mother was found to carry the c.258+ $2 \mathrm{~T}>\mathrm{C}$ mutation, whereas the father was heterozygous for three previously described polymorphisms, namely c.129162TTGGGGGTAAGAAAdelinsGGGGGCGGGGG and c. $129-71 \mathrm{G}>\mathrm{A}$ in intron 1 , and c. $201 \mathrm{~A}>\mathrm{G}$ in exon $2[7,10]$. The detection of a causal mutation associated with SDS and the compatible clinical picture prompted the study of the mRNA of the patient. cDNA analysis of the fragment encompassing exons 2 to 5 revealed the presence of two bands, one apparently normal sized and the other smaller than expected and that was not detected in the control sample (Fig. 2A). Sequencing of the patient's amplicons showed that those in the first band had an 8-bp deletion, known to be the expected product of the c.258+ $2 \mathrm{~T}>\mathrm{C}$ mutation, whereas those in the smaller band were missing the entire exon 3. The absence of exon 3 in the transcript could be explained either by abnormal splicing due to a mutation in one of the flanking introns, or by a gross genomic deletion.

Investigation at the gDNA level ruled out aberrant splicing, since only the allele carrying the c. $258+2 \mathrm{~T}>\mathrm{C}$ mutation was detected, upon intron analysis. Long-range gDNA PCR encompassing exons 2 to 4 revealed an additional band that was smaller than expected (normal fragment $3662 \mathrm{bp}$; variant fragment $\sim 2790 \mathrm{bp}$ ), which was also present in the patient's father (Fig. 2B). Nested PCR and sequencing enabled the characterization of the genomic breakpoints, disclosing a deletion of $872 \mathrm{bp}$, which included the entire exon 3 and part of the flanking intronic sequences. This alteration, characterized as c. $258+374 \_459+250 \mathrm{del}$, represents a new in-frame deletion that translates into a protein missing an internal 67 amino acids (p.Ile87_Gln153del), and hence most of its central domain.

In the context of genomic breakpoint analysis, large stretches of homologous sequences were identified in introns 2 and 3. Further investigation led to the conclusion that these were both AluSx repeats, with the deletion breakpoints embedded therein (Fig. 2C).

\section{Discussion}

SDS is a rare inherited multisystemic disorder characterized by pancreatic insufficiency, bone marrow dysfunction and skeletal changes. It may however involve other organs making diagnosis difficult. SDS exceptionally presents with severe anemia, as in this child [21], and on the other hand, MRC disorders are far more frequent than SDS [22,23]. Pearson syndrome was thus initially considered in this child, since the non-specific gastrointestinal, hepatic and hematological symptoms are commonly found in MRC disorders, to variable extents [24]. Diagnosis of MRC disorders is difficult and in children it is still frequently based on functional studies. The absence of a pathogenic mitochondrial or nuclear DNA 
A

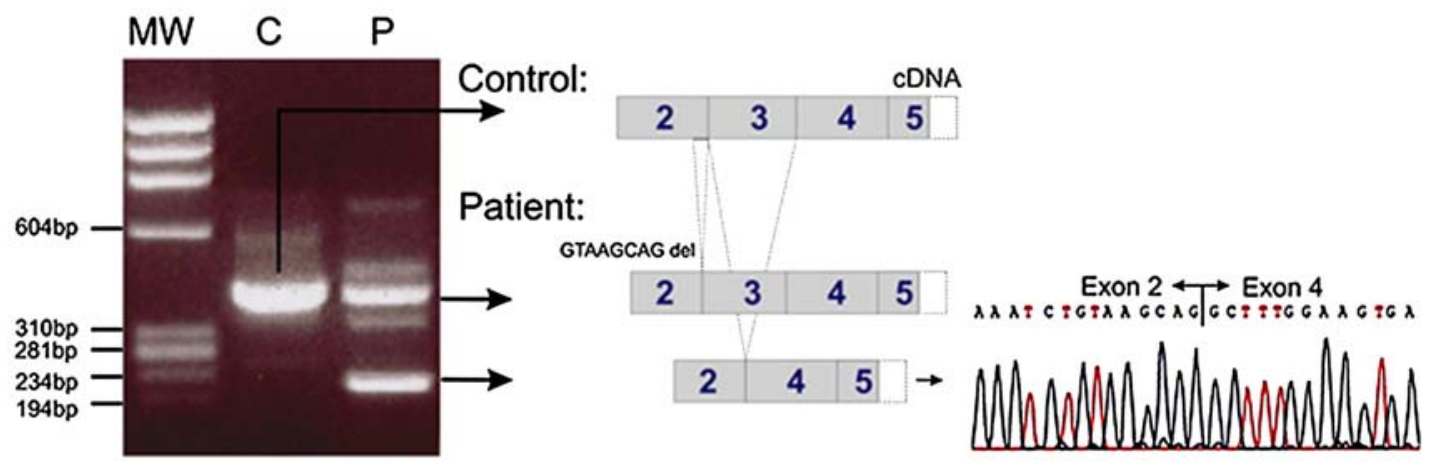

B

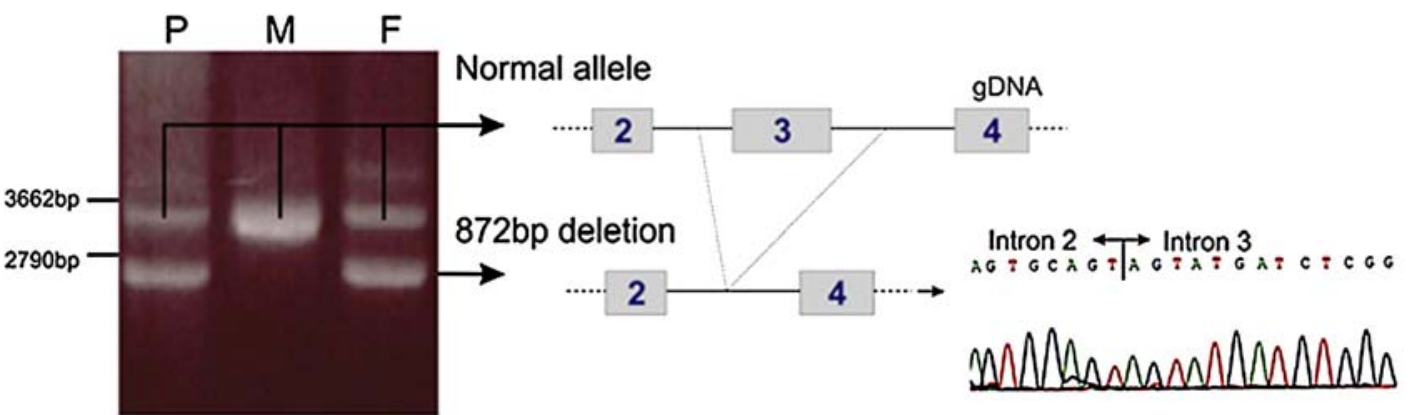

C

\begin{tabular}{|c|c|c|c|c|}
\hline Alus $x$ & (intron2) & 1 ...Exon 3 . & 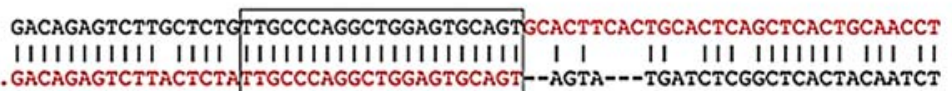 & 64 \\
\hline Alusx & $\begin{array}{l}\text { (intron } 2) \\
\text { (intron3) }\end{array}$ & 65 & 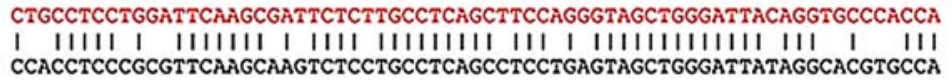 & 133 \\
\hline $\begin{array}{l}\text { Alus } \\
\text { Alusx }\end{array}$ & $\begin{array}{l}\text { (intron2) } \\
\text { (intron3) }\end{array}$ & 139 & 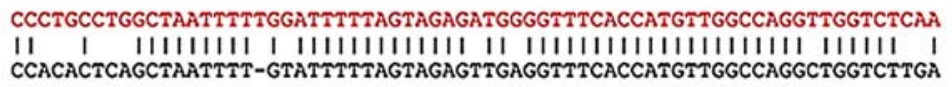 & 207 \\
\hline Alusx & (intron2) & 208 & 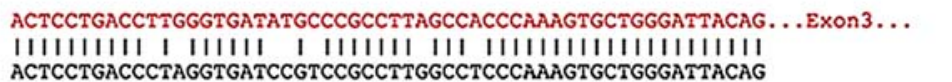 & 263 \\
\hline
\end{tabular}

Fig. 2. Molecular characterization of the novel deletion. (A) cDNA analysis of amplicons representing exons 2 to 5 . The patient presented two bands; one of almost normal size, but with an 8-bp deletion associated with the c.258+2T>C mutation, and the other missing the whole of exon 3. (B) gDNA analysis of amplicons representing exons 2 to 4. Long-range PCR revealed heterozygosity for the c.258+374_459+250del mutation, in the patient and in her father. (C) Alignment of AluSx repeats in introns 2 and 3. The sequence deleted in the new mutation is shown in red. The box highlights a 21-bp tract of perfect homology which marks the deletion breakpoints. MW—-molecular weight marker; $\mathrm{C}$ - control; $\mathrm{P}$ - patient; $\mathrm{M}$ - mother; $\mathrm{F}$ - father.

mutation is not a definitive criterion of exclusion, and therefore doubt remains as to the primary versus secondary nature of the MRC dysfunction. In this patient, MRC deficiency would be secondary, as favored by the observed reversal of liver mitochondrial dysfunction after prednisone administration. Besides the absence of the common mitochondrial DNA deletion, the typical skeletal dysplasia finally led to the clinical diagnosis of SDS.

In the molecular analysis of the SBDS gene, the presence of a hotspot region in and around exon 2 has facilitated diagnosis, and direct sequencing of this region has enabled the detection of at least one mutated allele in about $90 \%$ of SDS patients ([5], personal data). In the present case, the relatively frequent intron
2 mutation c. $258+2 \mathrm{~T}>\mathrm{C}$ was detected in heterozygosity, and gDNA sequencing of all other exons and exon-intron boundaries failed to reveal further alterations.

The c. $258+2 \mathrm{~T}>\mathrm{C}$ mutation is predicted to disrupt the donor splice site consensus dinucleotide sequence of intron 2, with subsequent use of an upstream cryptic donor splice site at position c.251_252GT. The resulting 8-bp deletion at the end of exon 2 in the respective transcript was described by Boocock et al. [7] and was also seen to be the only product of this mutation in the present case.

Extended cDNA analysis in our patient led to the identification of a second aberrant transcript, seen to be lacking exon 3 . Sequential analysis at the gDNA level enabled the detection of a 
gross deletion, characterized as c. $258+374 \_459+250 \mathrm{del}$. At the protein level, this in-frame exonic deletion is predicted to result in the loss of 67 amino acids (p.I87_Q153del), which comprises approximately $30 \%$ of the SBDS protein. In this region around $21 \%(14 / 67)$ of the residues are conserved or semiconserved, when comparing the representative orthologs across a diversity of species [8]. Recently, structural analysis of the SBDS homologue from Archaeoglobus fulgidus (AF0491) revealed a three-domain protein, where the central domain (residues 87160) was predicted to adopt a common fold - the winged helix-turn-helix (wHTH) [8]. Such HTH domains are widely used in DNA-binding and RNA-binding proteins of lower eukaryotes, but may also, to some extent, be involved in protein-protein interactions [8]. In the context of the human SBDS protein, this domain corresponds to residues $98-169$, a region which is almost completely abolished by the in-frame deletion described in the present study (residues 87-153). One may thus speculate that the resulting mutant SBDS protein will reflect the consequence of incorrect polypeptidic folding and concomitant loss of function.

Unlike most mutations described to date, which are attributed to conversion events resulting from the recombination between $S B D S$ and $S B D S P$ [7-13], in the present case the deletion is likely to have arisen from the mispairing of homologous sequences and unequal crossing over between introns 2 and 3 of the SBDS gene. When comparing the nucleotide sequence of these introns, we found stretches of $263 \mathrm{bp}$ in intron 2 and $257 \mathrm{bp}$ in intron 3 exhibiting approximately $80 \%$ homology. Further in silico analysis identified these as Alu sequences with AluSxsubfamily consensus.

The breakpoints of the deletion were found to coincide with the AluSx elements, and more specifically, within a 21-bp tract of perfectly homologous sequence near their $5^{\prime}$ ends (Fig. 2C). The fact that one copy of these tracts was deleted in its entirety, in the junction sequence, provides strong evidence for Alu-mediated homologous recombination as the mechanism involved.

Since Alu sequences are known to account for recurrent mutations $[25,26]$, we believe it may be advantageous to screen for deletional rearrangements involving exon 3 of the SBDS gene. This may be particularly useful in clinically compatible SDS cases where only one deleterious mutation is detected by routine screening methods. In such cases, cDNA analysis and/or Western blotting [9] would enable the detection of gross rearrangements in general. This information should shed light not only on the frequency (and recurrence rate) of the Alumediated exon 3 deletion, but also provide evidence for or against the monogenic nature of SDS.

An important implication in the failure to detect gross heterozygous deletions is that the identification of a single variant sequence may be erroneously interpreted as a homozygous mutation, if masked by a coincident exonic deletion. Moreover, the absence of the variant in one of the parents may be mistaken as evidence of a de novo occurrence.

Accurate molecular diagnosis is important not only for patient management, but also for genetic counseling of family members. As such, it is now increasingly recognized that screening for exonic rearrangements should be implemented in the routine diagnostic setting of many disorders, and our case illustrates how this might be extensive to SDS.

\section{References}

[1] H. Shwachman, L.K. Diamond, F.A. Oski, K. Khaw, The syndrome of pancreatic insufficiency and bone marrow dysfunction, J. Pediatr. 65 (1964) 645-663.

[2] M. Wilschanski, E. Hoeven, J. Phillips, B. Shuckett, P. Durie, Shwachman-Diamond syndrome presenting as hepatosplenomegaly, J. Pediatr. Gastroenterol. Nutr. 19 (1994) 111-113.

[3] D.R. Mack, G.G. Forstner, M. Wilschanski, M.H. Freedman, P. Durie, Shwachman syndrome: exocrine pancreatic dysfunction and variable phenotypic expression, Gastroenterology 111 (1996) 1593-1602.

[4] H. Ginzberg, J. Shin, L. Ellis, J. Morrison, W. Ip, et al., Shwachman syndrome: phenotypic manifestations of sibling sets and isolated cases in a large patient cohort are similar, J. Pediatr. 135 (1999) 81-88.

[5] S. Goobie, M. Popovic, J. Morrison, et al., Shwachman-Diamond syndrome with exocrine pancreatic dysfunction and bone marrow failure maps to the centromeric region of chromosome 7, Am. J. Hum. Genet. 68 (2001) 1048-1054.

[6] M. Popovic, S. Goobie, J. Morrison, et al., Fine mapping of the locus for Shwachman-Diamond syndrome at 7q11, identification of shared disease haplotypes, and exclusion of TPST1 as a candidate gene, Eur. J. Hum. Genet. 10 (2002) 250-258.

[7] G.R.B. Boocock, J.A. Morrison, M. Popovic, et al., Mutations in SBDS are associated with Shwachman-Diamond syndrome, Nat. Genet. 33 (2003) 97-101.

[8] A. Savchenko, N. Krogan, J.R. Cort, et al., The Shwachman-BodianDiamond syndrome protein family is involved in RNA metabolism, J. Biol. Chem. 280 (2005) 19213-19220.

[9] M.K. Austin, R.J. Leary, A. Shimamura, The Shwachman-Diamond SBDS protein localizes to the nucleolus, Blood 106 (2005) 1253-1258.

[10] E. Nakashima, A. Mabuchi, Y. Makita, et al., Novel SBDS mutations caused by gene conversion in Japanese patients with Shwachman-Diamond syndrome, Hum. Genet. 114 (2004) 345-348.

[11] O. Makitie, L. Ellis, P.R. Durie, et al., Skeletal phenotype in patients with Shwachman-Diamond syndrome and mutations in SBDS, Clin. Genet. 65 (2004) 101-112.

[12] J.R. Wolosznek, R.J. Rothbaum, A.S. Rawis, P.J. Minx, R.K. Wilson, P.J. Mason, M. Bessler, D.C. Link, Mutations of the SBDS gene are present in most patients with Shwachman-Diamond syndrome, Blood 104 (2004) 3588-3590.

[13] E. Nicolis, A. Bonizzato, B.M. Assael, M. Cipolli, Identification of nove mutations in patients with Shwachman-Diamond syndrome, Hum. Mutat. 25 (2005) 410.

[14] M. Erdos, K. Alapi, I. Balogh, G. Oroszlan, E. Rakoczi, J. Sumegi, L. Marodi, Severe Shwachman-Diamond syndrome phenotype caused by compound heterozygous missense mutations in the SBDS gene, Exp. Hematol. 34 (2006) 1517-1521

[15] H. Taneichi, H. Kanegane, T. Futatani, et al., Clinical and genetic analyses of presumed Shwachman-Diamond syndrome in Japan, Int. J. Hematol. 84 (2006) 60-62.

[16] R.M. Lima, E. Costa, C. Rocha, et al., Molecular characterization of a Portuguese patient with Shwachman-Diamond syndrome, J. Pediatr. Gastroenterol. Nutr. 41 (2005) 115-116.

[17] J.D. Thompson, T.J. Gibson, F. Plewniak, F. Jeanmougin, D.G. Higgins, The ClustalX windows interface: flexible strategies for multiple sequence alignment aided by quality analysis tools, Nucleic Acids Res. 25 (1997) 4876-4882.

[18] C. Burge, S. Karlin, Prediction of complete gene structures in human genomic DNA, J. Mol. Biol. 25 (268(1)) (1997) 78-94.

[19] T.A. Tatusova, T.L. Madden, BLAST 2 sequences, a new tool for comparing protein and nucleotide sequences, FEMS Microbiol. Lett. 174 (1999) 247-250

[20] J. Jurka, V.V. Kapitonov, A. Pavlicek, P. Klonowski, O. Kohany, J. 
Walichiewicz, Repbase update, a database of eukaryotic repetitive elements, Cytogenet. Genome Res. 110 (2005) 462-467.

[21] T.W. Kuijpers, E. Nannenberg, M. Alders, R. Bredius, R.C.M. Hennekan, Congenital aplastic anemia caused by mutations in the $S B D S$ gene: a rare presentation of Shwachman-Diamond syndrome, Pediatrics 114 (2004) 387-391.

[22] D. Skladal, J. Halliday, D.R. Thorburn, Minimum birth prevalence of mitochondrial respiratory chain disorders in children, Brain 126 (2003) 1905-1912.

[23] Y. Dror, Shwachman-Diamond syndrome, Pediatr. Blood Cancer 45 (2005) 892-901.
[24] A. Munnich, A. Rötig, V. Cormier, P. Rustin, Clinical presentation of respiratory chain deficiency, in: C.R. Scriver, A.L. Beaudet, W.S. Sly, D. Valle (Eds.), The Metabolic and Molecular Bases of Inherited Disease, 8th ed., McGraw-Hill, New York, 2001, pp. 2261-2274.

[25] Y. Koda, M. Soejima, P.H. Johnson, E. Smart, H. Kimura, An Alumediated large deletion of the FUT2 gene in individuals with ABOBombay phenotype, Hum. Genet. 106 (2000) 80-85.

[26] F. Ringpfeil, A. Nakano, J. Uitto, L. Pulkkinen, Compound heterozygosity for a recurrent $16.5-\mathrm{kb}$ Alu-mediated deletion mutation and single-basepair substitutions in the $A B C C 6$ gene results in pseudoxanthoma elasticum, Am. J. Hum. Genet. 68 (2001) 642-652. 